Braz J Med Biol Res, July 2010, Volume 43(7) 657-662

doi: 10.1590/S0100-879X2010007500050

Motor behavioral abnormalities and histopathological findings of Wistar rats inoculated with HTLV-1-infected MT2 cells

C.C. Câmara, R.B. Oriá, T.C. Felismino, A.P. da Silva, M.A.M. da Silva, J.V.A. Alcântara, S.B.C. Costa, A.C.P. Vicente, T.J. Teixeira-Santos and C.M. de Castro-Costa

The Brazilian Journal of Medical and Biological Research is partially financed by
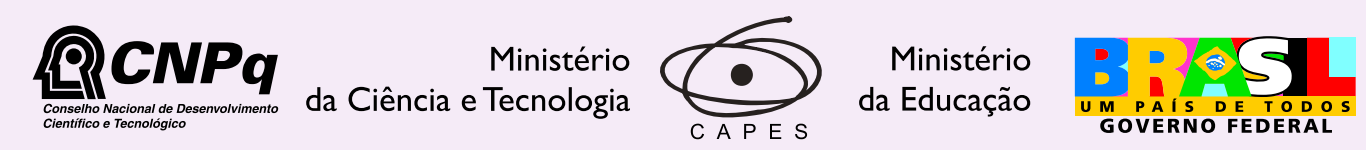

DIAPESP

Institutional Sponsors
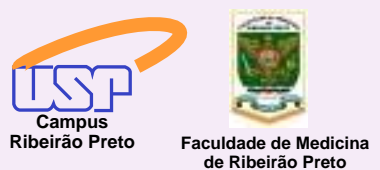

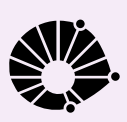

UNICAMP $\oplus$ SHIMADZU

GE Healthcare
Hotsite of proteomics metabolomics developped by: 


\title{
Motor behavioral abnormalities and histopathological findings of Wistar rats inoculated with HTLV-1-infected MT2 cells
}

\author{
C.C. Câmara ${ }^{1,2}$, R.B. Orián ${ }^{1,3}$, T.C. Felismino1 ${ }^{1}$, A.P. da Silva ${ }^{1}$, M.A.M. da Silva ${ }^{1}$, \\ J.V.A. Alcântara ${ }^{1}$, S.B.C. Costa ${ }^{1,3}$, A.C.P. Vicente ${ }^{4}$, T.J. Teixeira-Santos ${ }^{1,3}$ \\ and C.M. de Castro-Costa ${ }^{1,3, \dagger}$
}

${ }^{1}$ Laboratório de Neurologia Experimental e Neurofisiologia, Departamento de Fisiologia e Farmacologia, Faculdade de Medicina, Universidade Federal do Ceará, Fortaleza, CE, Brasil

2 Universidade Federal Rural do Semi-Árido, Mossoró, RN, Brasil ${ }^{3}$ Instituto de Biomedicina do Semiárido Brasileiro, Institutos Nacionais de Ciência e Tecnologia, Conselho Nacional de Desenvolvimento Científico e Tecnológico, Fortaleza, CE, Brasil

${ }^{4}$ Laboratório de Genética Molecular de Microorganismos, Departamento de Genética, Fundação Oswaldo Cruz, Rio de Janeiro, RJ, Brasil

\begin{abstract}
The objective of the present study was to describe motor behavioral changes in association with histopathological and hematological findings in Wistar rats inoculated intravenously with human T-cell lymphotropic virus type 1 (HTLV-1)-infected MT2 cells. Twenty-five 4-month-old male rats were inoculated with HTLV-1-infected MT2 cells and 13 control rats were inoculated with normal human lymphocytes. The behavior of the rats was observed before and 5, 10, 15, and 20 months after inoculation during a $30-\mathrm{min} /$ rat testing time for 5 consecutive days. During each of 4 periods, a subset of rats was randomly chosen to be sacrificed in order to harvest the spinal cord for histopathological analysis and to obtain blood for serological and molecular studies. Behavioral analyses of the HTLV-1-inoculated rats showed a significant decrease of climbing, walking and freezing, and an increase of scratching, sniffing, biting, licking, and resting/sleeping. Two of the $25 \mathrm{HTLV}-1$-inoculated rats ( $8 \%$ ) developed spastic paraparesis as a major behavioral change. The histopathological changes were few and mild, but in some cases there was diffuse lymphocyte infiltration. The minor and major behavioral changes occurred after 10-20 months of evolution. The long-term observation of Wistar rats inoculated with HTLV-1-infected MT2 cells showed major (spastic paraparesis) and minor motor abnormalities in association with the degree of HTLV-1-induced myelopathy.
\end{abstract}

Key words: Motor behaviors; HTLV-1-infected MT2 cells; Wistar rats; Experimental myelopathy

\section{Introduction}

Human T-cell lymphotropic virus type 1 (HTLV-1) is a widespread retrovirus that infects $10-20$ million people worldwide. HTLV-1 infection is associated with a range of nonmalignant chronic inflammatory disorders, of which the tropical spastic paraparesis/HTLV-1 associated myelopathy (TSP/HAM) is the best recognized, involving chronic progressive myelopathy characterized by spastic paraparesis, sphincter dysfunction, and mild sensory disturbance in the lower extremities (1).

An animal model of HTLV-1-associated myelopathy has been targeted by different research groups interested in offering a reliable tool for understanding the dynamics of the disease and related therapeutic approaches. Most models rely on targeted cell line studies and clinical expression of the condition (2). This clinical condition has been studied mostly in rats and rabbits infected with a variety of HTLV-1 strains.

Minagawa et al. (3) infected rabbits with two HTLV-1 strains isolated from a TSP/HAM patient from Colombia and from a seropositive asymptomatic blood donor, respectively. Of these rabbits, 6 developed persistent infection and became seropositive. Of four intracerebroventricularly and

Correspondence: C.C. Câmara, Biomedicina, UFC, Rua Cel. Nunes de Melo, 1315, 60430-270 Fortaleza, CE, Brasil.

Fax: +55-85-3366-8333. E-mail: mcastro@ufc.br

†/n memoriam

Received October 29, 2009. Accepted May 18, 2010. Available online June 7, 2010. Published July 9, 2010. 
intravenously inoculated rabbits, only 1 developed spasticity and hyperreflexia of the hind paw 6 weeks post-inoculation, while the remaining three rabbits showed transient and non-progressive or constant hyperreflexia.

Ishiguro et al. (4) inoculated rats with MT2 cells and the infectivity was found in more than $80 \%$ of them regardless of strain and age. However, anti-HTLV-1 antibody titers differed among strains and were significantly influenced by age at the inoculation time. The inoculated newborn rats remained seronegative, whereas adult rats (inoculated 16 weeks after birth) became seropositive. Three of 16 seronegative WKAstrain rats developed spastic paraparesis of the hind paw. Histopathologically, these animals showed spinal lesion with both myelin and axon damage in the lateral and anterior funiculi and massive macrophage infiltration predominating in the thoracic cord as well as the cervical and lumbar regions.

Kushida et al. (5) inoculated adult female WKA rats intravenously with Ra-1 and MT2 cells. One of 2 WKA rats inoculated with Ra- 1 cells at the age of 4 weeks developed HAM 27 months post-inoculation; however, of 8 F344 rats inoculated with MT2 cells at the age of 4 weeks, none developed HAM. The HAM rats were serologically positive.

In a previous study (6), the offspring of 18 Wistar rats inoculated with whole blood from a TSP/HAM patient showed a significant decrease in motor capacity (minor changes) and two of them exhibited spastic paraparesis (major changes). They were, however, HTLV-negative by serology. In the present study, we intended to reproduce HTLV-1-associated myelopathy by inoculating Wistar rats with HTLV-1-infected MT2 cells in order to identify minor and major motor behavioral abnormalities and to correlate them with possible histological changes.

\section{Material and Methods}

\section{Animals and treatment}

Twenty-five 4-month-old male Wistar rats weighing 300 to $350 \mathrm{~g}$ were housed in the vivarium of the Federal University of Ceará with free access to water and food and with 12-h day/night cycles. The spontaneous behaviors of the animals were recorded 1 week before inoculation with HTLV-1-infected MT2 cells at the dose of $2.6 \times 10^{6}$ cells/rat administered through a vein of the penis. The MT2 cells were kindly provided by Prof. Olindo Assis Martins Filho (Fiocruz, Belo Horizonte, MG, Brazil). This experimental group initially consisted of 25 rats and this number decreased with postinoculation time (5th month: 23 rats; 10th month: 23 rats; 15th month: 12 rats, and 20th month: 6 rats) as the animals were sacrificed for histopathological analysis or died due to unknown causes (the latter: $2 / 25=8 \%$ ). The animals were identified with numbers ranging from 20 to 27,30 to 37 , and 40 to 48 . This project was approved by the Committee of Animal Ethics of the Department of Physiology and Pharmacology at the Federal University of Ceará, and the number of animals used was kept to a minimum.
The control group (inoculated with normal human lymphocytes) initially consisted of 13 rats, with this number decreasing to 12 (5th month), 11 (10th month), 6 (15th month), and 6 (20th month). These rats were identified with numbers ranging from 1 to 7 and from 10 to 15 and were housed as described above.

\section{Behavioral studies}

For the behavioral analyses, cohort animals were kept in a wooden cage $(100 \times 50 \times 50 \mathrm{~cm})$ for a 5-min acclimation time. The observing cage (Plexiglas) was slightly illuminated, with the floor covered with wood shavings, and was placed in a dark and silent room. Positioned in front of the cage the observer could identify each behavioral component and record it using a computer software $\left(\right.$ Comporta $\left.^{\circledR}\right)$ designed by Prof. Marcus Vale (Federal University of Ceará, Fortaleza, CE, Brazil). Each experimental and control rat was observed for the following behaviors: scratching, sniffing, climbing, walking, freezing, licking, resting/sleeping, and biting. Each rat was observed for 30 min over a period of 5 consecutive days during the 5th, 10th, 15th, and 20th month post-inoculation. For measurements (in seconds), we considered the following spontaneous behaviors: i) scratching: time spent raising the hind paw to scratch parts of the body with rapid movements of the paw and claws; ii) sniffing: time spent probing the snout along and around surfaces with discrete head movements; iii) climbing: time spent climbing a 2-cm high three step ladder; iv) walking: time spent in lateral sequence gait with no period of free-flight (all four legs off the ground); v) freezing: time spent in a frozen position (absence of gross body movement); vi) licking: time spent grooming with the tongue; vii) resting/sleeping: time spent with closed eyes in the sleeping position; viii) biting: time spent piercing the skin with the teeth.

\section{Histopathological and biochemical analyses}

For histopathological analyses, the animals were randomly stratified as follows: 10 animals were sacrificed on the 10th month post-inoculation (numbers 32, 35, 40, 41, $42,43,45,46,47$, and 48), 6 animals on the 15th month post-inoculation (numbers 20, 24, 27, 30, 36, and 37), and 6 animals on the 20th month post-inoculation (numbers 21, 22, $23,25,26$, and 31 ). Subject number 33 was sacrificed during the 12th month post-inoculation due to extreme paralysis. For histopathological analysis, spinal cord samples from the cervical, thoracic and lumbar regions were embedded in paraffin and stained with hematoxylin-eosin.

Blood and spleen samples were taken from some animals for serological and molecular studies of HTLV-1 infection, respectively. Serological and PCR analyses were conducted at the Hemocenter of Ceará (Hemoce) and Oswaldo Cruz Foundation (Fiocruz) laboratories, respectively.

\section{Molecular studies}

The presence of anti-HTLV-1/2 antibodies was deter- 
mined by ELISA in duplicate (Abbott Murex HTLV-1/2, GE 80/81, Murex Diagnostics, UK). After serum screening, splenic blood from experimental rats were subjected to DNA extraction (QIAamp DNABlood Mini kit, Qiagen, Germany), according to manufacturer instructions. Proviral DNA was amplified by nested PCR with primers directed at the tax and long terminal repeat regions of the HTLV-1 genome.

\section{Statistical analysis}

Graph Pad Prisma ${ }^{\circledR}$ software was used for statistical analysis. Data are reported as means \pm SEM. Data were first analyzed by the Kolmogorov-Smirnov test to determine whether they had normal distribution and either unpaired Student $t$-test was used to compare means of two groups with normal distribution. When appropriate, the MannWhitney test was used to compare means of two groups with non-normal distribution (non-parametric data). The level of significance was set at $P<0.05$ in all analyses.
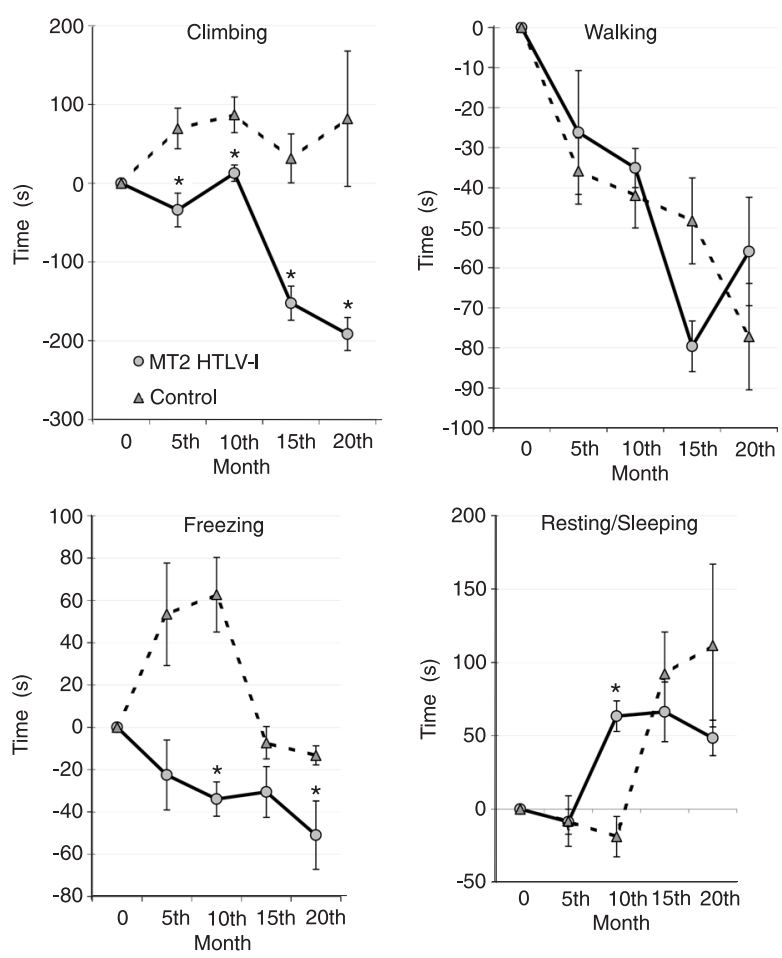

Figure 1. Significant $\left({ }^{*} P<0.05\right)$ decrease in the spontaneous behaviors climbing, walking, freezing, and resting/sleeping in 23 Wistar rats inoculated with HTLV-1-infected MT2 cells along 20 months of observation. Data are reported as mean delta values (time post-inoculation minus baseline behavior before inoculation) \pm SEM (unpaired Student $t$-test). HTLV-1 = human T-cell lymphotropic virus type 1 . Number of rats varied along the study, as follows (control/experimental): 5 th month $=12 / 23$ rats; 10 th month $=11 / 23$ rats; 15 th month $=6 / 12$ rats, and 20th month $=$ $6 / 6$ rats).

\section{Results}

\section{Behavioral studies}

The behavioral observations of the experimental rats showed a significant $(P<0.05)$ decrease of the following behaviors: climbing, walking, freezing, according to mean delta values from baseline (prior to inoculation). The resting/sleeping behavior was increased (Figure 1). We found reductions in the average time spent climbing among the experimental rats during overall testing, but especially by the 15th and 20th months post-inoculation $(P=0.0002$ and $P=0.001$, respectively). In addition, we found a significant decline in walking behavior only by the 15th month, with later recovery. On the other hand, there was a significant increase of scratching, biting (by the 20th month), licking (by the 15th month), and sniffing, with prolonged increases (from the 10th to the 20th month) in the experimental mice compared to controls $(P<0.05)$, as shown in Figure 2.
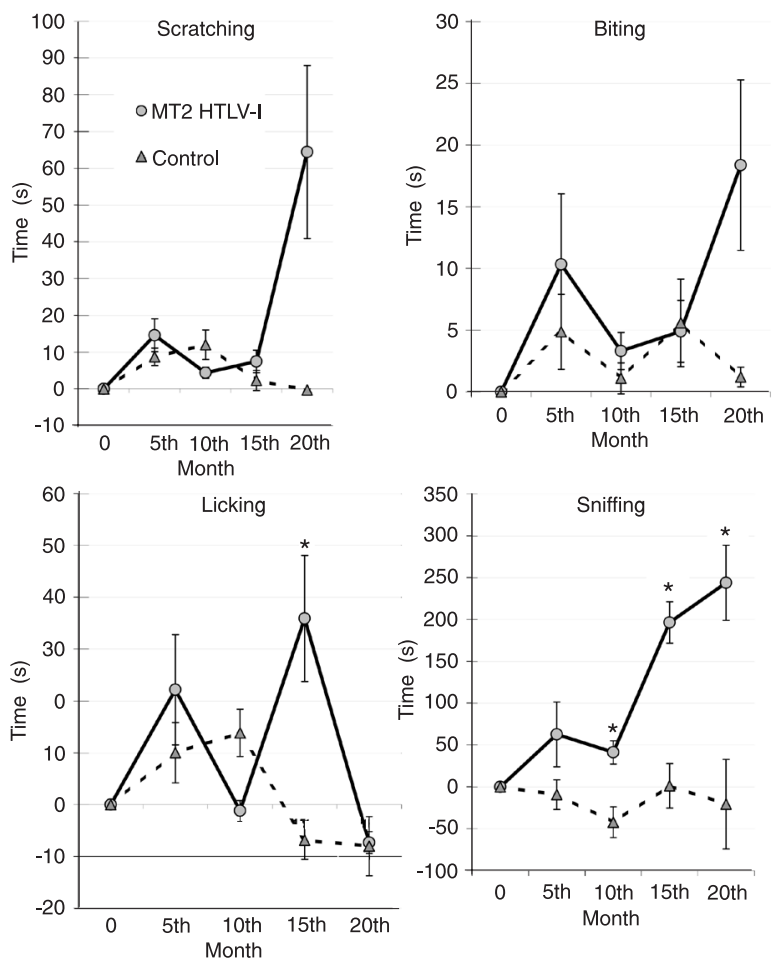

Figure 2. Significant $\left({ }^{*} P<0.05\right)$ increase in the spontaneous behaviors scratching, biting, licking, and sniffing in 23 Wistar rats inoculated with HTLV-1-infected MT2 cells along 20 months of observation. Data are reported as mean delta behavior values (experimental time post-inoculation minus baseline behavior before inoculation) \pm SEM (unpaired Student $t$-test). HTLV-1 = human T-cell lymphotropic virus type 1 . Number of rats varied along the study, as follows (control/experimental): 5 th month $=12 / 23$ rats; 10 th month $=11 / 23$ rats; 15 th month $=6 / 12$ rats, and 20th month $=6 / 6$ rats $)$. 
Among these rats, the following complementary abnormalities should be pointed out: rat No. 26 (experimental group) developed spastic paraparesis after 20 months of evolution (Table 1 and Figure $3 \mathrm{~A}$ and B). Rat No. 33 (experimental group) developed extreme spastic paraplegia precociously by the 12th month post-inoculation (Table 1 and Figure 3C). Rat No. 36 (experimental group) developed leukocytosis after the 15th month, with minor motor abnormalities. The details regarding the behavior, histopathology and clinical evolution of each rat are outlined in Table 1.

Furthermore, among the $25 \mathrm{HTLV}-1$-inoculated rats, 12 (48\%) presented motor abnormalities after 15 months of evolution and two (8\%) of them showed spastic paraparesis by 12 and 21 months after inoculation, respectively. Four (16\%) rats (Nos. 26, 33, 43, and 45) exhibited histopathological changes: 2 of them (Nos. 43 and 45) showed mild, non-inflammatory changes in the anterior horn of the spinal cord, and 2 rats (Nos. 26 and 33) were paraparetic and paraplegic, respectively, and exhibited loss and size reduction of neurons (Figure $4 \mathrm{~A}, \mathrm{~B}$ and $\mathrm{C}$ ), but only the spinal cord from rat No. 33 showed reactive gliosis (Table 1 and Figure 4D). Three rats (Nos. 22, 24 and 27) showed a reduction of leukocytes and 1 rat (No. 36) exhibited leukocytosis (Table 1).

The serological (ELISA) and PCR results of some rats inoculated with HTLV-1-infected MT2 cells were negative, possibly due to differences in viral loads and technical procedures.

\section{Discussion}

Despite the negative serology and PCR, the results of the analyses of the HTLV-1-inoculated Wistar rats showed minor and major motor abnormalities, corroborating previous results $(4,6)$. It is noteworthy that previous studies also did not show any seroconversion following HTLV-1 inoculation (4), likely due to variations in viral load and immunity responses affecting viral replication. In addition, Pro-viral gene detection by PCR may be possible only after prolonged infection (more than 20 months after inoculation)

Table 1. Summary of descriptive behavioral, histological, hematological, and evolutive data of Wistar rats inoculated with HTLV-1infected MT2 cells (experimental group; $N=23$ of the 25 that started the study).

\begin{tabular}{|c|c|c|c|c|}
\hline Rat & Behaviors & Histology & Hemogram & $\begin{array}{l}\text { Evolution } \\
\text { (months) }\end{array}$ \\
\hline 20 & Climbing and walking $\downarrow$ & Normal & Normal & 15 \\
\hline 21 & Climbing $\downarrow$ & Not done & Normal & 20 \\
\hline 22 & Climbing $\downarrow$ & Not done & Leukocytes $\downarrow$ & 20 \\
\hline 23 & Climbing $\downarrow$ & Not done & Normal & 15 \\
\hline 24 & Important climbing and walking $\downarrow$ & Normal & Significant leukocytes $\downarrow$ & 15 \\
\hline 25 & Significant climbing and walking $\downarrow$ & Normal & Normal & 15 \\
\hline 26 & Spastic paraparesis & Neuronal loss & Normal & 15 \\
\hline 27 & Climbing and walking $\downarrow$ & Normal & Leukocytes $\downarrow$ & 15 \\
\hline 30 & Important walking $\downarrow$ & Not done & Normal & 15 \\
\hline 31 & Important climbing and walking $\downarrow$ & Not done & Normal & 15 \\
\hline 32 & No changes & Normal & Normal & 10 \\
\hline 33 & Spastic paraplegia & Astrocyte proliferation and neuronal loss & Normal & 12 \\
\hline 35 & No changes & Not done & Normal & 10 \\
\hline 36 & Important walking $\downarrow$ & Normal & Leukocytosis & 15 \\
\hline 37 & Climbing and walking $\downarrow$ & Not done & Normal & 15 \\
\hline 40 & No changes & Normal & Normal & 10 \\
\hline 41 & No changes & Normal & Normal & 10 \\
\hline 42 & No changes & Normal & Normal & 10 \\
\hline 43 & Minimal motor changes & $\begin{array}{l}\text { Increased size of motor neurons of the anterior } \\
\text { horn of the inferior dorsal and lumbar cord (pre- } \\
\text { chromatolysis?) }\end{array}$ & Normal & 10 \\
\hline 45 & No changes & $\begin{array}{l}\text { Pre-chromatolytic anterior horn neurons of the } \\
\text { inferior dorsal and lumbar cord }\end{array}$ & Normal & 10 \\
\hline 46 & No changes & Normal & Normal & 10 \\
\hline 47 & No changes & Normal & Normal & 10 \\
\hline 48 & No changes & Normal & Normal & 10 \\
\hline
\end{tabular}

$\downarrow=$ decrease. HTLV-1 = human T-cell lymphotropic virus type 1. 
(7). Two (8\%) rats developed hind limb paralysis, a rate somewhat higher than the human prevalence of HTLV-1-associated myelopathy, which is only 0.25 to $5 \%$ of the infected population $(8,9)$. In human patients, the motor symptoms are broad and partial, impairing full gait, but not abolishing it completely, and only during the terminal phase, restricting the patients to wheelchairs or beds $(10,11)$. It is noteworthy that a similar finding was observed here, since 12 of 25 rats (48\%) had recognizable motor deficits, mainly concerning their ability to climb and walk. Freezing is a state of maintained contracted posture and the decrease of resting/sleeping is possibly a symptom of distress in this pathological condition. These motor abnormalities occurred after 15 months, as also shown by Minagawa et al. (3), Ohya (12) and Miyatake et al. (13), and with a peak after longer periods, such as 20 months, as shown by our results $(2,4,5)$. The period after 7 months has been pointed out as crucial for molecular events occurring in HAM rats $(4,14,15)$. Miyatake et al. (13) demonstrated the inhibitory effect of endogenous IFN- $\mathrm{Y}$ on the induction of the disease in non-WKA rats (like our animals), with a protective role against the development of myelopathy and infection.

On the other hand, the rats exhibited an increase of self-directed behaviors (scratching, biting and licking) and a decrease in resting/sleeping. Interestingly, licking was significantly increased earlier than scratching and biting behaviors, the latter probably associated with more physical distress. As shown by other investigators $(16,17)$, these increased behaviors could be an expression of distress such as pain. Further studies with pharmacological tests are needed to ascertain this finding in this experimental model, since pain has been described in human HTLV-1-associated myelopathy (De Castro-Costa CM, Câmara CC, Teixeira-Santos TJ, unpublished data).

The neuropathological analyses of our rats only demonstrated mild and nonspecific changes limited to the thoracic and lumbar spinal cord. Although lymphocyte infiltration was demonstrated in some rats, this finding was not observed universally, as also reported by others $(4,5)$. In addition, the hematological results were also nonspecific.

The present study has some gaps and limitations. However, its main purpose was to focus on minor behavioral changes shown in our previous study (6), correlating them with human clinical cases with incipient symptoms (18). In our previous study (6), we pointed out that the behavioral changes were observed in the offspring of Wistar rats inoculated with lymphocytes of HTLV-1-infected patients. The present study was carried out with Wistar rats inoculated with HTLV-1infected MT2 cells, and the results were similar in both studies. The evidence of minor motor changes may

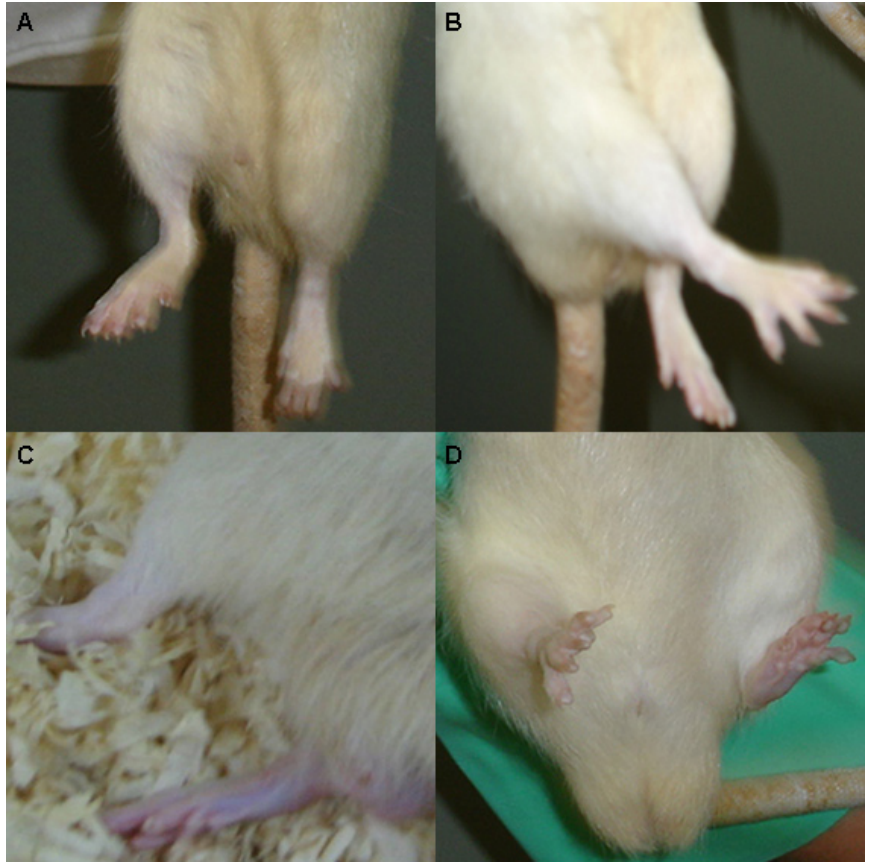

Figure 3. Ventral $(A)$ and lateral $(B)$ views of the hind limbs of the paraparetic rat (number 26) showing hyperextension. $C$, Dorsal view of the hind limbs of the paraplegic rat (number 33 ) showing hyperextension. $D$, Ventral view of the hind limbs of the control rat.
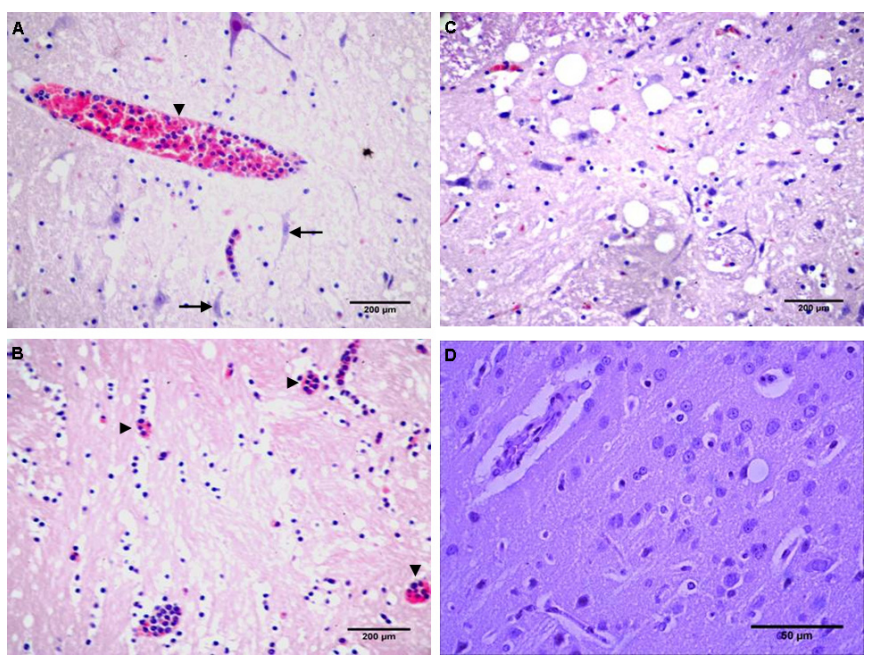

Figure 4. $A$, Spinal cord section of the paraparetic rat (number 26) showing anterior horn gray matter with decreased number and size of motor neurons (arrows) and a blood vessel congested with lymphocytes (arrowhead; HE, 100X). B, Spinal cord section of the paraparetic rat (number 26) showing anterior funiculus white matter, with diffuse lymphocyte infiltration from congested blood vessels (arrowheads; HE, 400X). Scale: $200 \mu \mathrm{m}$ for A and B. C, Lumbar spinal cord section of the paraplegic rat (number 33 ) showing the anterior horn with vacuolization and overall size reduction $(\mathrm{HE}, 100 \mathrm{X}) . D$, Astrocyte proliferation in the gray matter, with loss of neurons (HE, 400X). Scale: $200 \mu \mathrm{m}$ for $\mathrm{C}$ and $50 \mu \mathrm{m}$ for $\mathrm{D}$. 
be useful to follow the evolution of the disease, and, when possible, to conduct therapeutic trials with this model, since it may be more difficult to determine parameters in severely disabled animals with full paraplegia. These findings similarly support the human evolution of the disease with its expression during adult or older adult age, as observed here in rats aged 12 to 15 months, corresponding, on a human scale, to 40 to 60 years of age.

A molecular approach to these models has been extensively used by other investigators (19-21) and the present findings may complement these studies from a clinical

\section{References}

1. Saito M. Immunogenetics and the pathological mechanisms of human T-cell leukemia virus type 1- (HTLV-1-) associated myelopathy/tropical spastic paraparesis (HAM/TSP). Interdiscip Perspect Infect Dis 2010; 2010: 478461.

2. Costa CM, De Figueiredo EG, Santos TJ, Frota CH, Lobo CC, Santos FJ, et al. Experimental HTLV-I infection and associated myelopathy. Arq Neuropsiquiatr 1998; 56: 494497.

3. Minagawa H, Mora CA, Asher DM, Stone GA, Liberski PP, Gibbs CJ Jr. Transmission of human T-cell leukemia virus type I from a patient with HTLV-I associated myelopathy/ tropical spastic paraparesis and an asymptomatic carrier to rabbits. Arch Virol 1991; 118: 235-245.

4. Ishiguro $N$, Abe M, Seto K, Sakurai $H$, Ikeda $H$, Wakisaka A, et al. A rat model of human T lymphocyte virus type I (HTLV-I) infection. 1. Humoral antibody response, provirus integration, and HTLV-I-associated myelopathy/tropical spastic paraparesis-like myelopathy in seronegative HTLV-I carrier rats. J Exp Med 1992; 176: 981-989.

5. Kushida S, Matsumura M, Tanaka H, Ami Y, Hori M, Kobayashi $M$, et al. HTLV-1-associated myelopathy/tropical spastic paraparesis-like rats by intravenous injection of HTLV-1-producing rabbit or human T-cell line into adult WKA rats. Jpn J Cancer Res 1993; 84: 831-833.

6. De Castro-Costa CM. A preliminary analysis of an experimental long-term study of motor behavior and clinical aspects of Wistar rats inoculated with blood from HTLV-1 positive TSP patients from Ceará (Northeastern Brazil). In: Zaninovic' V (Editor), HTLV - Truths and Questions. Cali: Feriva; 1996. $p$ 89-95.

7. Suga T, Kameyama T, Kinoshita T, Shimotohno K, Matsumura M, Tanaka $\mathrm{H}$, et al. Infection of rats with HTLV-1: a small-animal model for HTLV-1 carriers. Int J Cancer 1991; 49: 764-769.

8. Uchiyama T. Human T cell leukemia virus type I (HTLV-I) and human diseases. Annu Rev Immunol 1997; 15: 15-37.

9. Araujo $A Q$, Leite AC, Lima MA, Silva MT. HTLV-1 and neurological conditions: when to suspect and when to order a diagnostic test for HTLV-1 infection? Arq Neuropsiquiatr 2009; 67: 132-138.

10. De Castro Costa CM, Carton H, Goubau P, D'Almeida JA. Brazilian studies on tropical spastic paraparesis. A metaanalysis. Arq Neuropsiquiatr 1994; 52: 585-591.

11. Olindo S, Cabre P, Lezin A, Merle H, Saint-Vil M, Signate point of view. Further analyses with better technical control regarding the diagnostic tests are warranted.

\section{Acknowledgments}

The authors acknowledge Dr. Olindo A. Martins Filho who kindly provided the MT2 cells and gave us important support in dealing with them, the Fundação Cearense de Apoio ao Desenvolvimento Científico e Tecnológico (FUNCAP) for support, and Mr. Sílvio A. Costa for preparation of this manuscript.
A, et al. Natural history of human T-lymphotropic virus 1-associated myelopathy: a 14-year follow-up study. Arch Neurol 2006; 63: 1560-1566.

12. Ohya O. [Chronological analyses of neuropathological and molecular biological changes in affected spinal cord of HTLV-I-infected rat (HAM rat disease)]. Hokkaido Igaku Zasshi 2000; 75: 117-134.

13. Miyatake $Y$, Ikeda H, Ishizu A, Baba T, Ichihashi T, Suzuki A, et al. Role of neuronal interferon-gamma in the development of myelopathy in rats infected with human T-cell leukemia virus type 1. Am J Pathol 2006; 169: 189-199.

14. Tomaru U, Ikeda H, Ohya O, Abe M, Kasai T, Yamasita I, et al. Human T lymphocyte virus type I-induced myeloneuropathy in rats: implication of local activation of the $\mathrm{pX}$ and tumor necrosis factor-alpha genes in pathogenesis. $J$ Infect Dis 1996; 174: 318-323.

15. Jiang $\mathrm{X}$, Ikeda $\mathrm{H}$, Tomaru U, Morita K, Tanaka Y, Yoshiki T. A rat model for human $T$ lymphocyte virus type I-associated myeloneuropathy. Down-regulation of bcl-2 expression and increase in sensitivity to TNF-alpha of the spinal oligodendrocytes. J Neuroimmunol 2000; 106: 105-113.

16. De Castro-Costa M, Gybels J, Kupers R, Van Hees J. Scratching behaviour in arthritic rats: a sign of chronic pain or itch? Pain 1987; 29: 123-131.

17. Kupers RC, Nuytten D, De Castro-Costa M, Gybels JM. A time course analysis of the changes in spontaneous and evoked behaviour in a rat model of neuropathic pain. Pain 1992; 50: 101-111.

18. De Castro-Costa CM, Araujo AQ, Barreto MM, Takayanagui OM, Sohler MP, da Silva EL, et al. Proposal for diagnostic criteria of tropical spastic paraparesis/HTLV-I-associated myelopathy (TSP/HAM). AIDS Res Hum Retroviruses 2006; 22: 931-935.

19. Tomaru U, Ikeda H, Jiang X, Ohya O, Yoshiki T. Provirus expansion and deregulation of apoptosis-related genes in the spinal cord of a rat model for human T-lymphocyte virus type I-associated myeloneuropathy. J Neurovirol 2003; 9: 530-538.

20. Lairmore MD, Silverman L, Ratner L. Animal models for human T-lymphotropic virus type 1 (HTLV-1) infection and transformation. Oncogene 2005; 24: 6005-6015.

21. Zhao TM, Hague B, Caudell DL, Simpson RM, Kindt TJ. Quantification of HTLV-I proviral load in experimentally infected rabbits. Retrovirology 2005; 2: 34. 